\title{
Abstracts of the 9th Annual Meeting of the British Paediatric Cardiac Association, Southampton, UK, 19-20 November 1998
}

Epidemiology of childhood cardiomyopathy in Australia: results of a ten-year population based study

Daubeney P.E.F, Nugent A., Datis A.M., Wilkinson J.L., Weintraub R.G.

The National Australian Childhood Cardiomyopatby Study, Royal Children's Hospital, Melbourne, Australia

The incidence and age distribution of primary cardiomyopathy (CM) in childhood has nor been well defined despire the porentially severe nature of these conditions. The epidemiology of childhood CM was examined as part of the National Australian Childhood Cardiomyopathy Study. This is a population-based study of all children within Australia presenting at less than 10 years of age with CM between 1987-1996. Cases of secondary $\mathrm{CM}$ were excluded. Each of the 8 cardiac centres was visited and patients enrolled from local databases, echo log books, medical records searches and submissions from cardiologists. Data was acquired from direct review of case records and all available cardiac investigations. During the 10 year study period, there were 283 new patients with CN from an age-specific population of 2,532,400 giving an incidence of new cases of 1.12 per 100,000 population per year.

$\begin{array}{llllll}\text { Diagnosis } & \text { Cases } & \text { M: F } & 0-1 \text { years } & 1-5 \text { years } & 5-10 \text { years } \\ \text { Dilated CM } & 182 & 1: 1.2 & 116 & 48 & 18 \\ \text { Hypertrophic CM } & 79 & 1: 0.5 & 56 & 12 & 11 \\ \text { Restrictive CM } & 11 & 1: 1.2 & 2 & 5 & 4 \\ \text { Mixed CM } & 11 & 1: 0.4 & 6 & 4 & 1\end{array}$

Mean annual occurrences of dilated, hypertrophic, restrictive and mixed CMs per $100,000$ of population were 0.72 (range: $0.40-1.09), 0.31(0.16-0.51), 0.04(0-0.08)$ and $0.04(0-0.08)$ respectively. Positive family history was found in $31 / 182$ patients (17\%) with dilated and in 15/79 (20\%) with hypertrophic CM. Five year survival for dilated CM was $66 \%$ (95\%CI 57-75), for hypertrophic CM 84\% (74-93) and for restrictive CM $31 \%$ (2-59). In conclusion, the majority of childhood dilated and hypertrophic CM presents within the first 12 months of life. Early age at presentation may reflect a more severe disease process. The relatively high familial incidences may indicate that genetic factors are important in paediatric $\mathrm{CM}$. We recommend that all first degree relatives be screened. This information may serve as the basis for further studies involving morphological/clinical comparisons and planning for future transplantation requirements.

The anatomy of the ventricular septal arteries in normal and abnormal hearts Hosseinpour AR, Anderson RH, Ho SY

National Heart and Lung Institute, Royal Brompton Campus, Imperial College of Science, Technology and Medicine, University of London, London, UK

Surgical repair of congenitally malformed hearts frequently involves sutures or incisions in, or close to, the ventricular septum. Little, however, is known about the arrangement of the septal arteries relative to the septal structures.
In this study, we traced the course of septal perforating arteries in 46 hearts. We studied 6 hearts with isolated ventricular sepral defect (VSD), 10 with tetralogy of Fallot, 13 with double outlet right ventricle, 6 with double inlet left ventricle (DIIV), 6 with absent right atrioventricular connection (ARAVC) and 5 normal hearts. All hearts were dissected from the right ventricular aspect, and the arteries related to the medial papillary muscle, VSD and the outlet septum.

Overall, the first and/or second septal arteries were the largest ones. In the majority of the hearts, the site of origin of the first septal artery could by predicted by projecting a perpendicular line from the inferior wall of the heart, through the base of the medial papillary muscle, to the anterior descending coronary artery. Distally, the artery terminated at the base of the medial papillary muscle, unless it encountered a VSD or the insertion of an outlet septum. The former resulted in the termination of the vessel. The latter caused it to veer toward the ventricular apex. After clearing the outlet septum, the vessel either turned back to terminate at the base of the medial papillary muscle, or gave rise to one or two branches that did so. In hearts with DILV and ARAVC, the first septal artery approached the anterior margin of the VSD but, without the medial papillary muscle, its course was less predictable.

The medial papillary muscle and the outlet septum, where present, are landmarks for the course of the first septal artery.

A pathological mechanism for systemic right ventricular dysfunction in congenitally corrected transposition of the great arteries (ccTGA)

Homung TS, Bernard EJ. Celermajer DS, Howman-Giles RB, Hawker RE

Adolph Basser Cardiac Institute, New Children's Hospital, Sydney, Australia

Aims: To determine the pathological mechanism of right ventricular (RV) dysfunction in ccTGA and to assess the effectiveness of a drug therapy based on this mechanism. Background: In subjects with ccTGA, the morphological RV supplies the systemic circuit. This arrangement renders the RV susceptible to progressive failure: the pathological mechanisms of this are unclear.

Methods: 11] 20 patients with ccTGA, aged 3-34 (mean 15) years, had sestamibi myocardial perfusion scans at rest and at peak exercise. RV myocardial perfusion and function were assessed in 12 segments, perfusion being graded 0 (normal) to 3 (severely impaired). 'Perfusion score' was defined as the sum of the grades for all 12 segments. [2] 6 of these patients then underwent repeat sestamibi scanning at peak exercise, before and after administration of $5 x \mathrm{~g} / \mathrm{kg}$ of intravenous perindoprilat (ACE-inhibitor). Results: [1] Fixed RV perfusion defects were found at rest in all 20 patients, involving $4.6 \pm 2.3$ segments (of a total of 12 segments) per patient. $90 \%$ of affected areas also showed a concordant reduction in wall motion and/or thickening. Reversible defects were apparent after exercise in $17 / 20$ (85\%). RV ejection fraction (RVEF) was, $55 \%$ in nearly $2 / 3$ of the parients, mean RVEF being $52 \pm 12 \%$. Perfusion score at res' $\because$ i. an $6.5 \pm 3.2)$ correlated inversely with RVEF ( $p=0.05$ ). [2] After perindoprilat, systulic BP fell by $10 \pm 7 \mathrm{mmHg}$. There was, however, no consistent change in perfusion, with improvement in only $0.3 \pm 3.2$ segments per parient and in perfusion score of $0.2 \pm 3.2$ $(\mathrm{p}=\mathrm{NS})$. 
Conclusions: These data suggest that ischaemia/infarction secondary to inadequate myocardial perfusion underlies the development of lare RV dysfuncrion in cCTGA. A lack of improvement after perindoprilat, however, suggests that ACE-inhibition is unlikely to alter this process.

Intermittent perfusion during deep hypothermic circulatory arrest (DHCA) results in normal cerebral hemodynamic, metabolic and ultrastructural recovery Langley SM, Chai PJ. Miller SE, Mault JR, Jaggers JJ. Lodge AJ. Ungerleider RM Soutbampron, UK and Duke University Medical Center, Durham, North Carolina, USA

DHCA can be useful for repair of some heart defects in infants, but has been shown to cause an impairment in recovery of cerebral blood flow (CBF) and cerebral metabolism (CMRO, that is proportional to the duration of the DHCA period. This effect on $\mathrm{CMRO}_{2}$ may be a marker for brain injury, since $\mathrm{CMRO}_{2}$ recovers normally following cardiopulmonary bypass (CPB) when DHCA is not used. Our aims with this study were twofold. First, to investigate the effects of intermittent perfusion during DHCA in the anticipation that it would not result in significant impairment to $\mathrm{CMRO}$, following $\mathrm{CPB}$ and secondly, to correlate these findings with electron microscopy of the cerebral microcirculatory bed (EM).

Fifteen neonatal piglets were placed on $\mathrm{CPB}$ and cooled to $18^{\circ} \mathrm{C}$. Each animal then underwent either: 1). 60 minutes (min) continuous $\mathrm{CPB}$ (control); 2), 60 min uninterrupted DHCA (U1-DHCA); or 3) $60 \mathrm{~min}$ DHCA with intermittent perfusion $(1 \mathrm{~min}$ every $15 \mathrm{~min}$ ) (I-DHCA). All animals were then rewarmed and weaned from CPB. Measurements of $\mathrm{CBF}$ and $\mathrm{CMRO}_{2}$ (radioactive Xe clearance) were taken before and after CPB. A further 9 animals underwent CPB withour DHCA (2 animals) or with DHCA ( 7 animals), under various conditions of arterial blood gas management, intermitrent perfusion and reperfusion time.

UI-DHCA resulted in significant impairment to recovery of $\mathrm{CMRO}_{2}$ following $\mathrm{CPB}$ $(p<0.05)$. Regardless of the blood gas strategy used, the EM following UI-DHCA revealed extensive damage characterised by perivascular intracellular and organell edema, and vascular collapse. I-DHCA, on the other hand, produced a pattern of normal $\mathrm{CMRO}_{2}$ recovery identical to controls and the EM was normal for borh these groups.
Intermittent perfusion during DHCA is clinically practical and results in normal cerebral metabolic and ultrastructural recovery. Furthermore, the correlation between brain structure and $\mathrm{CMRO} 2$ suggests that monitoring $\mathrm{CMRO} 2$ in the operating room may be an ourstanding way to manage brain protection during infant heart surgery.

Substrate provision with $L$-arginine and endogenous NO-release reverse the pulmonary endothelial dysfunction after congenital heart surgery

Shulze-Neick 1, Penny DJ. Rigby $M L$, Morgan C. Kelleber A, Collins $P, L J$, Bush $A$, Shineboume EA, Redington AN

The Royal Brompton and Harefield NHS Trust, London, UK

Introduction: We examined pulmonary endothelial dysfunction (PED) in children with congenital heart disease by assessing the L-arginine-nitric-oxide pathway in terms of substrate provision (L-arginine [L-Arg]), endogenous nitric oxide (NO) release (substance $\mathrm{P}$ [Sub $\mathrm{P}$ ]), and inhalatory NO (inh NO) provision before and after open heart surgery.

Patients and Methods: 14 preoperative (PreOp) patients (age0.6-1.9 years) with pulmonary hypertension undergoing cardiac catheterisation, and 13 patients (age 0.6-1.5years) between 203 hour after cardiopulmonary bypass (PostOp) were examined. All were ventilated, sedated and paralyzed. Oxygen uptake was measured by respiratory mass spectrometry. Blood samples and pressure measurements were taken from catheters in the pulmonary artery and the pulmonary vein. Cardiac output was determined by the direct Fick method. Pulmonary vascular resistance index (PVRI) was calculated at $\mathrm{FiO}=0.21$ and 0.65 , and then during cumulative administration of intravenous $L-A r g$ and Sub $P$, and then inhNO.

Results: PreOp, there was no demonstrable PED after the administration of oxygen. PostOp, there was marked PED which was completely relieved with L-Arg and Sub P with no further effect of inhNO.

Conclusion: Both substrate deficiency and failure of endogenous NO-release appear to contribute to postoperative PED. These preliminary results may indicate new treatment strategies. 